\title{
Fuzzy-logic-RSSI based approach for cluster heads selection in wireless sensor networks
}

\author{
Azamuddin bin Ab Rahman, Mohd Nizam Mohmad Kahar, Wan Isni Sofiah Wan Din \\ Faculty of Computer Systems \& Software Engineering, Universiti Malaysia Pahang, Malaysia
}

\begin{abstract}
Article Info
Article history:

Received Jul 30, 2019

Revised Oct 29, 2019

Accepted Nov 13, 2019

\section{Keywords:}

Cluster head

Fuzzy logic

RSSI

Wireless sensor network

ABSTRACT

Wireless Sensor Networks (WSNs) are defined as networks of nodes that work in a cooperative way in order to sense and control the surrounding environment. Several WSNs algorithms have been proposed by utilizing the Fuzzy Logic technique to select the cluster heads ( $\mathrm{CHs}$ ). Each technique employs a different combination of input parameters such as nodes density, communication cost, and residual energy. $\mathrm{CHs}$ determination is critical towards this goal, whereas the combination of input parameters is expected to play an important role. Nevertheless, the received signal strength (RSSI) is one of the main criteria which get little attention from researchers on the topic of CHs selection. In this study, an RSSI based scheme was proposed which utilizes Fuzzy Logic approach in order to be combined with residual energy and centrality of the fuzzy descriptor. In order to evaluate the proposed scheme, the performance Multi-Tier Protocol (MAP) and Stable Election Protocol (SEP) were compared. The simulation results show that the proposed approach has significantly prolonged the survival time of the network against SEP and MAP, while effectively decelerating the dead process of sensor nodes.
\end{abstract}

Copyright $@ 2020$ Institute of Advanced Engineering and Science. All rights reserved.

\section{Corresponding Author:}

Azamuddin Abdul Rahman,

Faculty of Computer Systems \& Software Engineering,

Kuantan, Pahang, Malaysia.

Email: azamuddin@ump.edu.my

\section{INTRODUCTION}

The Internet of Things (IoT) is an emerging topic among the computer network researchers that make computing truly ubiquitous. This development provides an opportunity to discover a novel technology that leads to long-range communication and conservation of the network energy simultaneously. As future IoT applications would require power efficient usage, wireless network transmissions should operate reliably and efficiently in order to be able to be utilized for long periods of time. Studies regarding wireless networks have evolved during the last decade with numerous deployments on wireless sensor networks (WSNs), mostly comprising tracking and monitoring applications [1-4].

Clustering technique was previously employed in order to achieve energy efficiency in WSNs. By means of clustering, the whole network is divided into several clusters, each having a cluster head $(\mathrm{CH})$. Sensor nodes in the cluster pass the information to its respective $\mathrm{CH}$, then the $\mathrm{CH}$ would aggregate and compress the received data to be forwarded to the BS. Numerous CHs selection algorithm based on different fuzzy logic input parameters were proposed in the literature [5-8]. The goal of the CHs selection algorithm is to minimize the energy consumption of sensor nodes. Combination of fuzzy logic input parameters enable efficient $\mathrm{CHs}$ selection and help in reducing the sensors battery consumption. For instance, work in [9] has improved upon LEACH based on residual energy, centrality, and a number of neighbouring nodes. The sensor nodes having the highest number of neighbours and the highest remaining energy are elected as $\mathrm{CH}$. The nearest distance between sensor nodes and $\mathrm{CHs}$ is negligible in this algorithm; hence it potentially 
drains sensor nodes energy even faster. Through exploring previous studies in the area of reduction of energy consumption, it can be observed that majority of efforts has been made towards CHs selection, while few studies have focused on noise and signal strength in a fuzzy descriptor perspective.

Building on this insight, an RSSI was proposed as a new input parameter to be combined with other parameters such as residual energy and centrality. Due to the complex task of CHs selection, the fuzzy logic tool was employed in order to determine the suitable nodes as CHs. The Fuzzy Logic can generalize probability and is able to deal with the approximate analysis from imprecise hypothesis and generating the conclusion [10]. Since the form of if-then rule fits in human reasoning, this inference process projects crisp quantification into human language and produces a precise value of output which could determine that which node should be selected as the $\mathrm{CH}$. The experiments demonstrated that the proposed combination of fuzzy descriptors is capable of reducing the energy by $40 \%$ through sacrificing the performance of the wireless network. Simulations results indicated that the RSSI is significant when it is considered as a fuzzy logic descriptor.

The rest of the paper is organized as follows. In Section 2, related studies were presented. Section 3 briefly discussed the model of the energy consumption scheme. Then, fuzzy-logic-RSSI-based scheme approach is proposed in Section 4. Next, in Section 5, the fuzzy logic approach was described, followed by the simulation settings in Section 6. Then, the experimental results were discussed in Section 7. Finally, Section 8 concludes this paper.

\section{RELATED WORKS}

The topic of WSNs is a technology with more than 60 years of development and standardization. First published in 1950, the original version of the WSN standard was proposed for military purposes known as Sound Surveillance System (SOSUS) [11]. The WSN is a collection of the connected sensors, embedding software and hardware through the base station to the Internet. The architecture of WSN consists of three main components known as sensor node, a base station (BS) or gateway, and user. It is commonly installed in the form of a cluster consist of a large number of sensor nodes to cover a wide area network.

The energy consumption of the nodes is utilized by three main components: the sensors, the transceivers, and the data transmission, while data transmission consumes the major amount of energy [12]. The data transmission process in WSNs requires the source and the sensor nodes to exchange data packets which lead to battery depletion. Accordingly, the node receiving data from all of the member nodes within the cluster is known as CHs. Then, $\mathrm{CHs}$ sent the aggregated data to the BS. Therefore, the selection of CHs essentially affects the WSNs lifetime. Commonly, ideal CHs are the ones which possess the highest amount of residual energy, while being in the nearest distance between sensor nodes and CHs. In fact, $\mathrm{CHs}$ itself consumes a lot of energy due to the activity of data transmission and processing. The $\mathrm{CH}$ selection is crucial towards energy efficiency and production of the best signal quality. In order to select a suitable $\mathrm{CH}$, it is imperative to appropriately set parameters for considerations. A fuzzy descriptor for $\mathrm{CH}$ selection has been investigated by many researchers [13-19]. In a study by [20], the authors employed residual energy as an input parameter, whereas such parameter is preferred by the researchers. Since residual energy has been utilized as an input parameter, the potential CHs would be selected with the highest remaining energy. In WSN environment, the values of sensors' energy are different due to the energy constrained condition [21].

In previous studies [17-19], RSSI was considered on the threshold value for CHs selection, without considering the Fuzzy Logic approach. In addition, these studies did not consider the path loss model in the RSSI calculation which is an important criterion which should be taken into account, as the distances of the nodes are different. RSSI was utilized as a range-based algorithm in localization technique for distance estimation between the nodes. Other common range based techniques are known as Time of Arrival (TOA) algorithm, Angle of Arrival (AOA), and Time Difference of Arrival (TDOA). In a study performed by Kannan et al. [18], CHs selection in 2-tier network based on residual energy and RSSI was presented. It was reported that the nodes which are close to the average RSSI value can be elected as CHs. Although the study considered the inter-cluster routing, the location of BS which was not centered with the nodes could result in inefficient use of energy.

\section{ENERGY MODEL}

Each sensor node is equipped with a single battery where it consumes energy in transmitting data packets, sensing, and processing. In order to evaluate the energy consumption in the network, some metrics have been proposed, as presented in [20]. The energy consumption model in data transmission is adopted 
from [21], in which the consumed energy of transmitter nodes greatly depends on the transmitted packet with (k) bits over the distance (d). It can be calculated as:

$$
E_{T X}(k . d)=\left\{\begin{array}{c}
k * E_{\text {elect }}+k * \varepsilon_{f S} * d^{2}, d \leq d_{o} \\
k * E_{\text {elect }}+k * \varepsilon_{m p} * d^{4}, d \geq d_{o}
\end{array}\right.
$$

where $E_{\text {elect }}$ is energy dissipated per (k) bit to run the transmitter, $\varepsilon_{f s}$ is the amplifier's energy consumption per bit during data transmission if the distance $\leq d_{o}, \varepsilon_{m p}$ is the amplifier's energy consumption per bit during data transmission if the distance $d \geq d_{o}$. Upon receiving the data, energy consumption is calculated as:

$$
E_{R X}=k * E_{\text {elect }}
$$

All the sensor nodes in the cluster communicate with their respective $\mathrm{CHs}$. Thus, the energy dissipates in the $\mathrm{CH}$ during transmission is given by:

$$
E_{C H}=\left(\frac{N}{L}-1\right) * k * E_{\text {elect }}+\frac{N}{L} * k * E_{D A}+k * E_{\text {elect }}+k * \varepsilon f s^{*} d^{2}
$$

where $\mathrm{L}$ is the number of clusters, $\mathrm{d}$ is the average distance between the $\mathrm{CH}$ and the $\mathrm{BS}, E_{D A}$ is the energy of data aggregation which is $5 \mathrm{~nJ} / \mathrm{bit} /$ signal, and $\mathrm{N}$ is the total number of sensor nodes. Note that the number of average node per cluster can be obtained by $\left(\frac{N}{L}-1\right)$ where -1 is a non-CH node.

\section{PROPOSED FUZZY LOGIC BASED RSSI SCHEME}

In this study, CHs selection was proposed based on an RSSI scheme by using a fuzzy logic method that involves a combination of three input parameters. Specifically, residual energy, centrality, and RSSI are the parameters that were considered in order to select CHs. Residual energy is the most important input parameters for $\mathrm{CH}$ selection. It indicates the remaining battery power of the sensor nodes. In other word, sensor nodes with high residual energy can process and transmit more data. Aimed at node centrality, it is the sum of the distances between the nodes and its neighbor. This measurement represents the centrality of the nodes to other nodes (i.e., $\mathrm{CH}$ ). In other words, centrality refers to the number of direct connections between a node and its neighbour. In order to obtain the centrality value, the sum distance from child node, i to the $\mathrm{CHs}, \mathrm{j}$ denoted as $d_{i, j}$ is calculated based on Euclidean distance as follow:

$$
\mathrm{d}_{i, j}=\sum \sqrt{\left(\left(x_{i}-x_{j}\right)^{2}+\left(y_{i}-y_{j}\right)^{2}\right)}
$$

where $\left(x_{i}, \mathrm{y}_{i}\right)$ is the position of child node, $\mathrm{i}$ and $\left(x_{j}, \mathrm{y}_{j}\right)$ is the position of $\mathrm{CH}, \mathrm{j}$ distributed in the network. Based on (4), centrality can be defined as:

$$
\operatorname{Centrality}\left(k_{y}\right)=\frac{\left(\sum_{y=1}^{k} d_{i, j}\right)}{k-1}
$$

where $k_{y}$ is the number of nodes in the network, $d_{i, j}$ represents the distance between node $\mathrm{i}$ and its $\mathrm{CH}, \mathrm{j} . d_{i, j}$ is equal to 0 when the node is a $\mathrm{CH}$. Based on this centrality value, it can be used to establish far, satisfactory and near average distance to the $\mathrm{CHs}$.

For that reason, the received signal strength limit should be below than the original noise floor to allow the sensor nodes to access the spectrum. In this case, the original noise floor was set to $10 \mathrm{dBm}$. The chance of the sensor nodes to become $\mathrm{CH}$ is low if the required signal energy falls under the acceptable and bad signal state. If the located nodes satisfy the associated conditions, the nodes with the strongest RSSI will be elected as CHs. Commonly, to evaluate the level of interference due to signal distortion, RSSI is used which denotes the received signal power loss [22]. The RSSI technique is suitable to be implemented to WSN because its implementation is cheap and complexity is low. RSSI is defined as the voltage measured by receivers received signal strength indicator. Often, it measures the power of the signal at the receiver based on the known transmit power. The log-distance path loss is used to include in RSSI measurement since it is suitable for the indoor and outdoor environment. The log-distance path loss model $P(l)$ is expressed as [23]: 


$$
P(l)=P_{o} \mathrm{X}\left(\frac{d_{o}}{d}\right)^{\alpha}+\mathrm{X}_{\sigma}
$$

where $P_{0}$ is the path loss at distance $d_{o}$ from the source and $\alpha$ is the path loss exponent. $\alpha$ is typically set as 2-6 depending on the environment (i.e., forest and indoor home). Thus, it was set as 3 to suit with an indoor and outdoor environment [24]. $\mathrm{d}$ is a distance between transmitter and receiver. $X_{\sigma}$ is a zero mean Gaussian that reflect the white Gaussian noise due to multi-path and shadow fading. This path loss measurement is in decibels $(\mathrm{dBm})$ unit.

\section{FUZZY LOGIC APPROACH}

Once in the fuzzifier stage, the crisp values such as residual energy, centrality, and RSSI are converted into fuzzy data or Membership Functions (MFs) where it indicates the intersection point of the value of the parameter degree between 0 and 1 . Three linguistic variables were defined for each membership function as shown in Figure 1-3. The linguistic states defined for membership function are as follows:

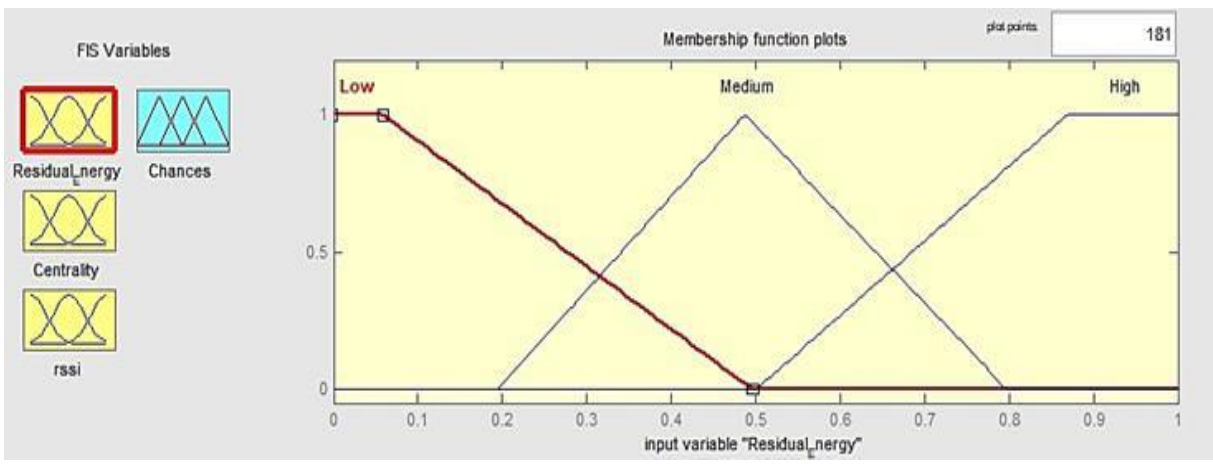

Figure 1. Membership Function of Residual Energy

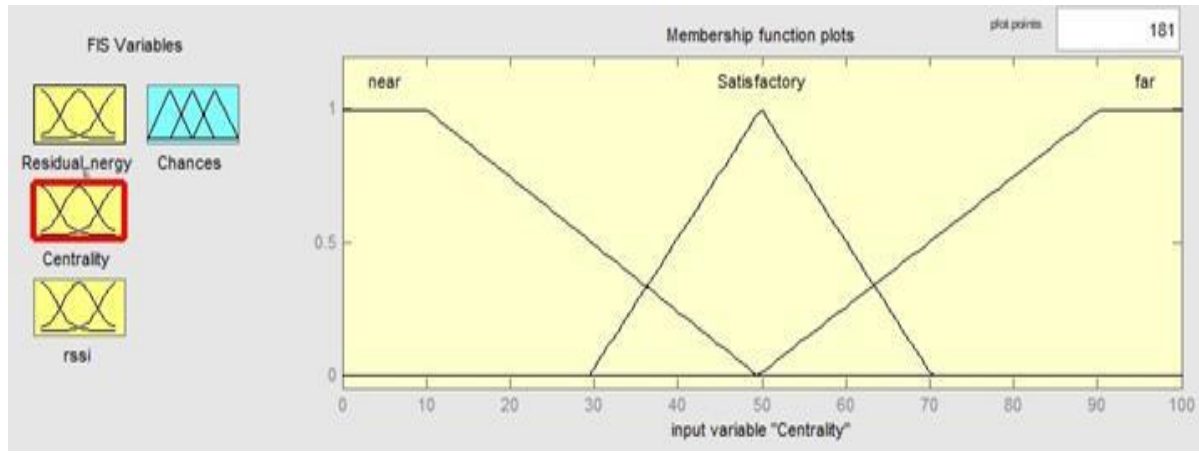

Figure 2. Membership Function of Centrality

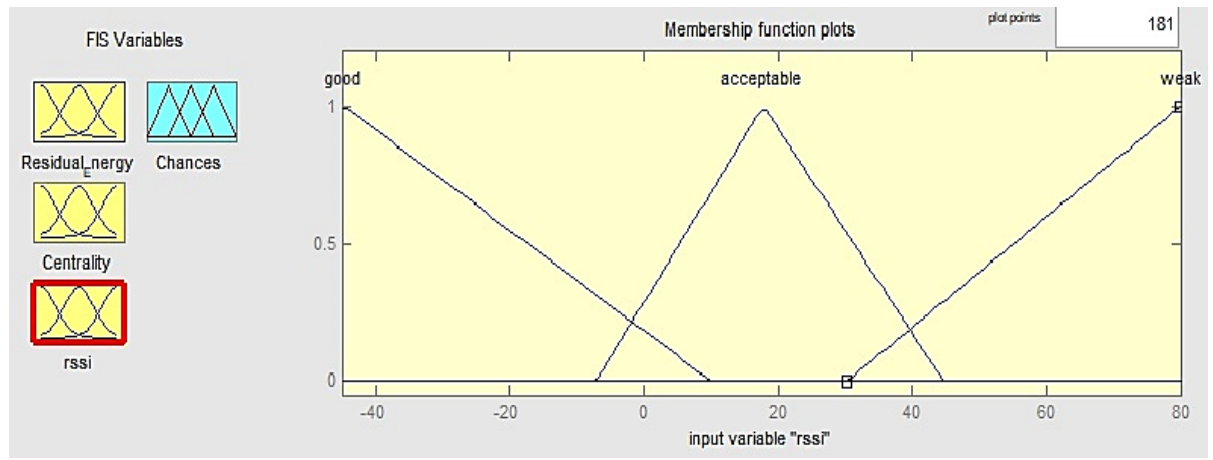

Figure 3. Membership Function of RSSI

Fuzzy-logic-RSSI based approach for cluster heads selection in wireless... (Azamuddin bin Ab Rahman) 
The value for residual energy is divided evenly into low, medium, and high that has an interval of 0 to $0.5,0.2$ to 0.8 , and 0.5 to 1 , respectively. The linguistic variable for centrality is divided into 0 to 50 for near, 30 to 70 for satisfactory, and 50 to 100 for far [25]. Designed for RSSI, the linguistic variables are divided into -45 to 10 for good, -10 to 45 for acceptable, and 30 to 80 for bad [5]. Subsequently, the second step is Rule Evaluation. In this step, the membership values obtained from the previous step will produce fuzzy output determined based on IF-THEN (fuzzy decision-making block) rules. The possible outcome for $\mathrm{CHs}$ selection is defined as low, medium, high. For instance, if the residual energy is high, centrality is near and RSSI is good, while the chance for the node to be considered as a $\mathrm{CH}$ is high. Algorithm 1 illustrates the proposed combination of fuzzy parameters for CHs selection.

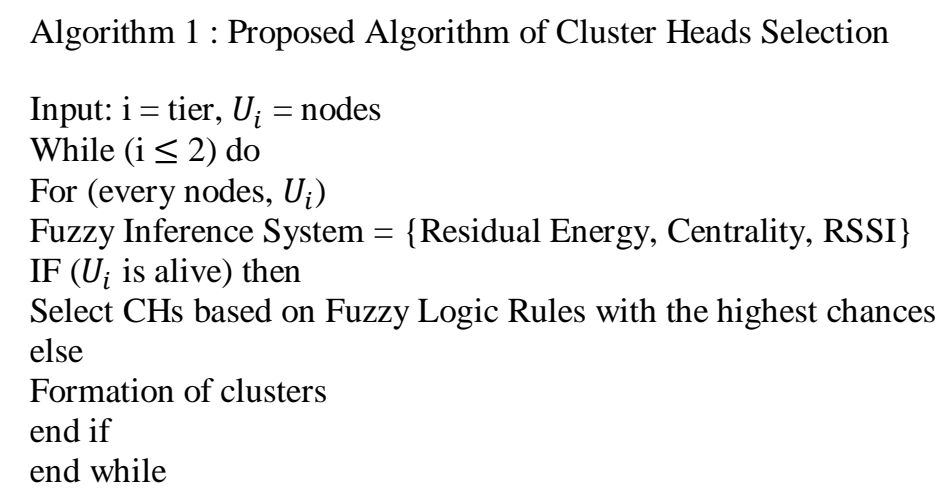

\section{SIMULATION SETTINGS}

The WSN network was simulated by employing MATLAB with the aim to evaluate its energy consumption. The performance is evaluated based on 3 performance metrics: First Dead Node (FDN), last Dead Node (LDN), and Total Dead Node (TDN). FDN is measured by the time required for the very first sensor nodes to die in the network, while LDN is the time for the last node to die in the network. Moreover, TDN is the number of the dead node after certain rounds. The proposed protocol known as Relay Access Protocol (RAP) protocol was compared with Stable Election Protocol (SEP) and MAP protocol. The simulation parameters setting in experiments are presented in Table 1.

\begin{tabular}{cc} 
Table 1. The Simulation & Parameter Settings \\
\hline Parameter & Value \\
\hline Sensor Nodes & 100 \\
Network Coverage & $100 \mathrm{~m} \times 100 \mathrm{~m}$ \\
Position of Base Station & Center $(50,50)$ \\
1-tier Radius & $25 \mathrm{M}$ \\
2-tier Radius & $50 \mathrm{M}$ \\
Energy (J) & $1 \mathrm{~J}$ \\
Number of bits & 8000 \\
\hline
\end{tabular}

\section{SIMULATION RESULTS}

The experiments were divided into several sets, while the first one observes the FDN and LDN that consists of a combination of input parameters i.e., ResCen, ResRSSI, and ResCenRSSI. Furthermore, performances of RAP with MAP and SEP in terms of FDN and LDN were evaluated. Afterward, the packet received by $\mathrm{CHs}$ and $\mathrm{BS}$ was analyzed for such combination parameter. Finally, total dead nodes between RAP, MAP, and SEP were analyzed.

Figure 4 shows the FDN and LDN for a combination of ResCen, ResRSSI, and ResCenRSSI input parameters. At the initial state, all the sensor nodes contained high energy which resulted in the existence of no dead nodes. After a while, nodes began to die in the 3711th, 3643th, and 3994th iterations for ResCen, ResRSSI, and ResCenRSSI, respectively. During this state, child nodes attempt to transmit data to their respective $\mathrm{CHs}$, and from $\mathrm{CH}$ s to the relay node, and from the relay node to $\mathrm{BS}$, which in fact would lead to energy depletion. Moreover, competition among sensor nodes for data transmission leads to energy loss as well. At around 5000th, it can be observed that the number of dead nodes is steadily maintained for all combinations of input parameters for a certain period, as the remaining number of alive nodes is low. Through the existence of the small number of sensor nodes, the reduction can be observed in the competition 
for data transmission, while the process of energy dissipation will be very slow. Until 5204th iteration, LND was first observed in ResRSSI followed by ResCen and ResCenRSSI at 5204th and 5222th iterations, respectively. The combination of ResCenRSSI has the best performance in terms of FND and LDN, since it considers the path loss of the nodes to be selected as CHs.

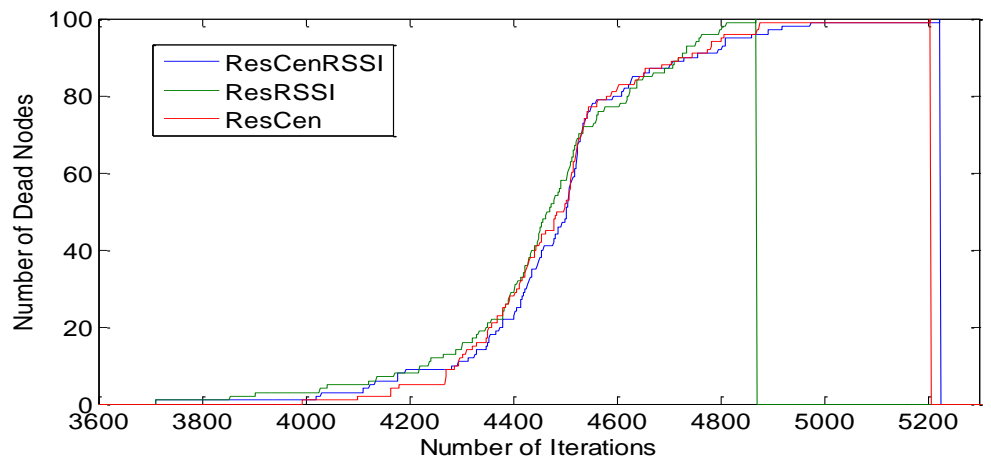

Figure 4. First Dead Node (FDN) and Last Dead Node (LDN) for Different Combination of Fuzzy Descriptor

Figure 5 illustrates the performance evaluation of RAP, MAP, and SEP in terms of FDN and LDN. It can be observed that the FDN for RAP and MAP have a slight alteration at 3993th and 3711th iterations, respectively. However, the earliest FDN for SEP was observed at 709th iteration. The LDN for RAP, MAP, and SEP were at 5222th, 5022nd, and 5010th iterations, respectively.

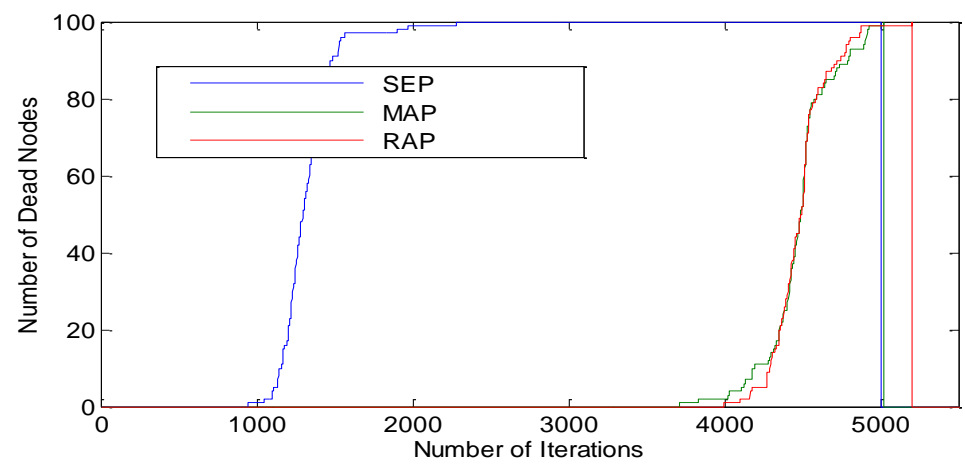

Figure 5. First Dead Node (FDN) and Last Dead Node (LDN) of RAP, MAP and SEP

According to the obtained results, the RAP protocol that utilizes RSSI based schemes for CHs selection has the best performance in terms of FND and LND. This is due to the fact that the proposed combination of input parameters selects the potential $\mathrm{CH}$ that has the strongest received signal which relatively depends on the centrality and residual energy of the nodes. The efficiency of ResCenRSSI was verified in this experiment which was observed to outperform the MAP and SEP by $18 \%$ and $35 \%$, respectively. MAP protocol utilizes three combination parameters known as residual energy, centrality, and communication cost. The performance of MAP is considered to be acceptable where it demonstrates the stability of dead nodes for the most period of iterations. In fact, the proposed RSSI input parameters in this study consider the communication cost when estimating the path loss of each sensor nodes as well, whereas the distance is a crucial element in the path loss equation. This indicates that the received signal strength was taking its effect, as the distances between nodes and BS became shorter, that could be more significant than the communication cost input parameter.

Figures 6 shows the average residual energy per iteration in the network when each protocol was run until the end of its lifetime. The energy consumption of an iteration comprised of the energy consumed during clustering formation and data transmission. The average residual energy decreased steadily although there were frequent changes of $\mathrm{CHs}$ due to the high number of sensor nodes. This proved that the proposed 
approach performed well with a high number of sensor nodes. The average residual energy of the protocol was determined by the $\mathrm{CHs}$ selection approach and data transmission strategy, which led to an energy consumption efficiency. Thus, it was obvious that the proposed relay communication and new combination of input parameters could greatly improve the energy efficiency of MAP, resulting in an energy cost decrease.

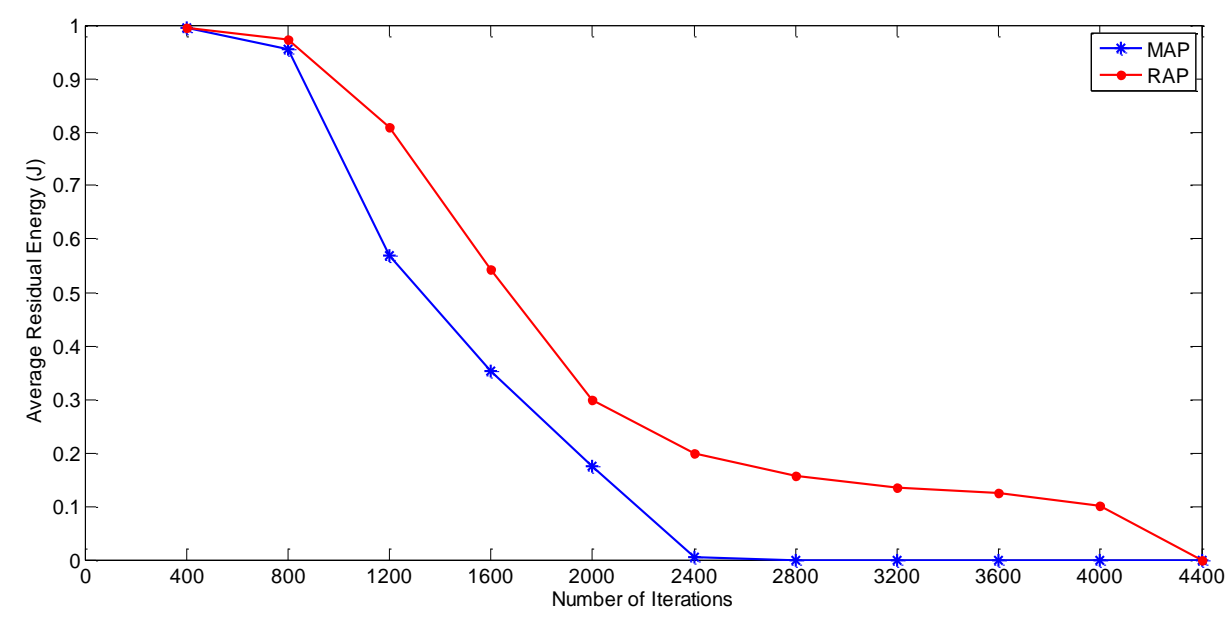

Figure 6. Average Residual Energy

\section{CONCLUSION}

In this study, three combinations of fuzzy input parameters known as residual energy, centrality, and RSSI were proposed. The RSSI was recommended in order to resolve the high energy consumption when $\mathrm{CHs}$ with low received signal strength are selected. Through simulations, it was demonstrated that the proposed combination of input parameters outperformed the ResCen and ResComm. In addition, the obtained results confirmed that integration of RSSI with residual energy and centrality input parameter through the utilization of Fuzzy approach is able to accurately estimate the chance of CHs, which could contribute to longer network lifetime as compared to SEP and MAP protocols. In conclusion, the experimental results obtained in this study could provide an insight on the feasibility of employing RSSI measurements in CHs selection.

\section{ACKNOWLEDGEMENTS}

The authors would like to thank UMP PGRS Grant (190391) for providing financial support and facilities. In addition, we would like to acknowledge and send our gratitude to the Faculty of Computer Systems \& Software Engineering and Systems Network \& Security UMP for their support.

\section{REFERENCES}

[1] Y. Li, C. S. Chen, J. Zhang, and K. Chi, "Optimal Relay Placement for WSN-Based Home Health Monitoring System," pp. 129-137, 2016.

[2] M. N. Ismail, M. A. Shukran, M. Rizal, M. Isa, M. Adib, and O. Zakaria, "Establishing a Soldier Wireless Sensor Network ( WSN ) Communication for Military Operation Monitoring," vol. 7, no. 2, pp. 89-95, 2018.

[3] T. Rault, A. Bouabdallah, and Y. Challal, "Energy efficiency in wireless sensor networks: A top-down survey," Comput. Networks, vol. 67, pp. 104-122, 2014.

[4] M. Srbinovska, C. Gavrovski, V. Dimcev, A. Krkoleva, and V. Borozan, "Environmental parameters monitoring in precision agriculture using wireless sensor networks," J. Clean. Prod., vol. 88, pp. 297-307, 2015.

[5] S. Amri, F. Khelifi, A. Bradai, A. Rachedi, M. L. Kaddachi, and M. Atri, "A new fuzzy logic based node localization mechanism for Wireless Sensor Networks," Futur. Gener. Comput. Syst., 2017.

[6] T. Shankar, S. Shanmugavel, and A. Rajesh, "Hybrid HSA and PSO algorithm for energy efficient cluster head selection in wireless sensor networks," Swarm Evol. Comput., vol. 30, pp. 1-10, 2016.

[7] P. Nayak and A. Devulapalli, "A Fuzzy Logic-Based Clustering Algorithm for WSN to Extend the Network Lifetime,” IEEE Sens. J., vol. 16, no. 1, pp. 137-144, 2016. 
[8] M. Toloueiashtian and H. Motameni, "A new clustering approach in wireless sensor networks using fuzzy system," J. Supercomput., vol. 74, no. 2, pp. 717-737, Feb. 2018.

[9] D. Jia, H. Zhu, S. Zou, and P. Hu, "Dynamic Cluster Head Selection Method for Wireless Sensor Network," IEEE Sens. J., vol. 16, no. 8, pp. 2746-2754, 2016.

[10] D. R. Das Adhikary and D. K. Mallick, "A fuzzy-logic based relay selection scheme for multi-hop wireless sensor networks," no. September, pp. 4-5, 2015.

[11] M. Kocakulak and I. Butun, "An overview of Wireless Sensor Networks towards internet of things," 2017 IEEE 7th Annu. Comput. Commun. Work. Conf., pp. 1-6, 2017.

[12] H. El Alami and A. Najid, "Energy-efficient fuzzy logic cluster head selection in wireless sensor networks," in 2016 International Conference on Information Technology for Organizations Development (IT4OD), 2016, pp. 1-7.

[13] O. S. Kwon, K. Jung, and J. Lee, "WSN Protocol based on LEACH Protocol using Fuzzy," vol. 12, no. 20, pp. 10013-10018, 2017.

[14] S. Rana, A. N. Bahar, N. Islam, and J. Islam, "Fuzzy Based Energy Efficient Multiple Cluster Head Selection Routing Protocol for Wireless Sensor Networks," Int. J. Comput. Netw. Inf. Secur., vol. 7, no. 4, pp. 54-61, 2015.

[15] S. Chhabra and V. Arora, "A Review On General Self-Organized Tree-Based Energy," vol. 16, no. 2, pp. 7591-7596, 2017.

[16] Y. K. Sharma and S. Kumar, "A Clusterhead Selection Technique for a Heterogeneous WSN and Its Lifetime Enhancement Using HeteroLeach Protocol," in Proceedings of the International Conference on Microelectronics, Computing \& Communication Systems, 2018, pp. 247-257.

[17] R. Yan, H. Sun, and Y. Qian, "Energy-aware sensor node design with its application in wireless sensor networks," IEEE Trans. Instrum. Meas., vol. 62, no. 5, pp. 1183-1191, 2013.

[18] G. Kannan and T. Sree Renga Raja, "Energy efficient distributed cluster head scheduling scheme for two tiered wireless sensor network," Egypt. Informatics J., vol. 16, no. 2, pp. 167-174, 2015.

[19] S. Fazackerley, A. Paeth, and R. Lawrence, "Cluster head selection using RF signal strength," Electr. Comput. Eng. 2009. CCECE '09. Can. Conf., no. July 2015, pp. 334-338, 2009.

[20] D. Agrawal and S. Pandey, "FUCA: Fuzzy-based unequal clustering algorithm to prolong the lifetime of wireless sensor networks," Int. J. Commun. Syst., vol. 31, p. e3448, 2017.

[21] B. Kan and L. Cai, “An A ccurate Energy Model for WSN Node and Its O ptimal Design,” no. 2006, pp. 328-332.

[22] J. Zheng, Y. Liu, X. Fan, and F. Li, "The Study of RSSI in Wireless Sensor Networks," vol. 133, no. 2, pp. 207-209, 2016.

[23] S. Kurt and B. Tavli, "Path-Loss Modeling for Wireless Sensor Networks: A review of models and comparative evaluations.," IEEE Antennas Propag. Mag., vol. 59, no. 1, pp. 18-37, 2017.

[24] J. Miranda et al., "Path loss exponent analysis in Wireless Sensor Networks: Experimental evaluation," 2013 11th IEEE Int. Conf. Ind. Informatics, no. July, pp. 54-58, 2013.

[25] S. A. Sahaaya Arul Mary and J. B. Gnanadurai, "Enhanced Zone Stable Election Protocol based on Fuzzy Logic for Cluster Head Election in Wireless Sensor Networks," Int. J. Fuzzy Syst., vol. 19, no. 3, pp. 799-812, Jun. 2017.

\section{BIOGRAPHIES OF AUTHORS}

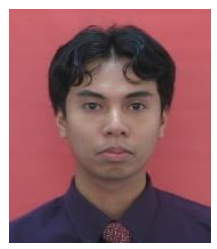

Azamuddin Ab Raman received Bachelor and Master Degree in Computer Science from University Utara Malaysia in 2012 and 2015 respectively. Since 2017, he has been pursuing his PhD degree in the Faculty of Computer System \& Software Engineering, University Malaysia Pahang, Malaysia. His current research interests include sensor networks, fuzzy logic, and wireless interference.

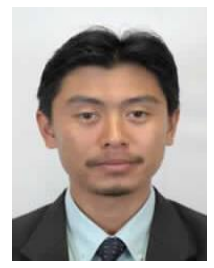

Mohd Nizam Mohmad Kahar received the PhD degree from Nottingham University, UK. He is currently senior lecturer with the Faculty of Computer System \& Software Engineering, University Malaysia Pahang, Malaysia. He has authored and co-authored over 100 refereed papers in journals, conferences, and workshop proceedings about his research areas within six years. His research interests include hybrid algorithm, scheduling, and time tabling. He is a member of the IEEE.

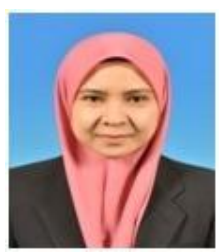

Wan Isni Sofiah Wan Din received the PhD degree from Universiti Teknologi Mara, Shah Alam in 2016. Since then, she has been a senior lecturer in the Faculty of Computer System \& Software Engineering, University Malaysia Pahang, Malaysia. She is currently investigating energy consumption of WSNs, drone technology and future IoT. 